\title{
On-line Interferometric Observation of Thermomechanically Induced Refractive Index Changes during Glass Welding by Ultra-short Laser Pulses
}

\author{
Kristian Cvecek ${ }^{1,2}$, Johannes Heberle ${ }^{2,3}$, Isamu Miyamoto ${ }^{2,4}$, Michael Bergler ${ }^{3,5}$, Dominique de Ligny ${ }^{5}$ and Michael \\ Schmidt ${ }^{1,2,3}$ \\ ${ }^{1}$ blz - Bayerisches Laserzentrum, Konrad-Zuse Str. 2-6, 91052, Erlangen, Germany \\ E-mail: k.cvecek@blz.org \\ ${ }^{2}$ Graduate School in Advanced Optical Technologies, Friedrich-Alexander-University of \\ Erlangen-Nürnberg, Paul-Gordan-Str. 6, 91052 Erlangen, Germany \\ ${ }^{3}$ Institute of Photonic Technologies, Friedrich-Alexander-University of Erlangen-Nürnberg, \\ Konrad-Zuse-Str. 3/5, 91052 Erlangen, Germany \\ ${ }^{4}$ Osaka University, 2-1 Yamada-Oka, Osaka 565-0871, Japan \\ ${ }^{5}$ Institute of Glass and Ceramics, Friedrich-Alexander-University of Erlangen-Nürnberg, \\ Martensstr. 5, 91058 Erlangen, Germany
}

\begin{abstract}
Glass welding by ultra-short puled lasers is known to exhibit a strongly localized heat affected zone, thereby allowing the fusion welding of glass parts with thermally sensitive components nearby. Moreover, the shape of the refractive index change induced by the thermomechanical gradients has an impact on the laser beam propagation into the molten zone and could cause e.g. an eventual focus shift or deteriorate the beam quality. While the temperature of the heat affected zone can be estimated by simulations, measurements of the Raman shift or thermography cameras, the shape of the temperature and stress gradients cannot be completely recovered by the aforementioned techniques due to specific limitations. In this work we present an interferometric approach to measure a 2dimensional change of the refractive index around the molten zone before, during and after welding. Moreover comparisons between phase distribution inside the cold and unmodified glass with the hot and modified as well as cold and modified glass make it possible to estimate the thermal and the stress-induced refractive index modifications around the molten zone separately.
\end{abstract}

DOI: 10.2961/jlmn.2018.03.0027

Keywords: pump-probe, glass, ultra-short pulsed lasers, refractive index, phase, welding, laser processing, thermal evolution

\section{Introduction}

Processing of glass by ultra-short pulsed (USP) lasers found its way into many industrial applications such as filament cutting [1], selective laser-induced etching [2] and also glass welding which was first introduced by [3] in 2006. Compared with the other process it is likely that an adequate description of glass welding requires the widest "cross-section" of different effects: The process is initiated by nonlinear absorption [4,5] (e.g. multi photon ionization) of the cold (room temperature) glass material which is fully transparent for the laser wavelength. Depending on the properties of the focusing optics and pulse duration also self-focusing effects may influence the shape of the focal spot generating free electrons [6]. Depending on the pulse duration and energy of the pulse the initially by purely nonlinear effects generated electrons can be accompanied by avalanche ionization [7] (still within the same pulse) which can dramatically increase the free electron density further and thereby the absorptivity of the irradiated region. The generated free electrons will transfer their kinetic energy subsequently to the glass lattice and heat the glass material. If the next pulse arrives "early" and overlapped enough with respect to the first pulse (by choosing a suitable pulse repetition rate and feed speed) so that the temperature of the glass has not returned back to room temperature the heat will accumulate in the focal region with each incoming pulse [8] until a quasi-static state is reached. The maximum achievable temperatures can lie in the range of few thousand degrees [8]. Nonetheless, even at comparably lower temperatures (several hundred ${ }^{\circ} \mathrm{C}$ ) free electrons can be generated by thermal ionization. Since plasma, i.e. free electrons, is typically an outstanding absorber $[8,10]$, the ionization process can take place far above the actual focus position as can be seen in Fig. 1 and was shown by simulations in [11]. Moreover, the plasma generated "upstream" of the incoming laser beam will shield the irradiation inside laser spot [12]. However, this situation is not stable resulting in quasi-periodic plasma movement of plasma generated in the focal spot moving towards the incoming laser beam until the local temperature becomes too low for a sufficient number of thermally ionized seed electrons for avalanche ionization, as has been shown by experiments $[11,13]$ and simulations [11]. Consequently, the temperature inside the melt and its surroundings has not only a large impact on the temperature-induced solid-liquid state changes (important for welding) but also on the ongoing absorption process of the laser by plasma. 
While there exist several attempts to study the temperature evolution during irradiation by simulations, certain values for e.g. the heat conductivity and capacity at high temperatures have to be assumed because there are no or insufficient data on these properties. Of course there have been attempts to measure the temperature distribution directly by Raman shift [14]. Unfortunately, if the measurements are conducted close to the molten zone or even inside, the intensity of the thermal radiation surpasses the desired signals for measurement. Furthermore, while the method is easy to implement for a measurement at a single spot, its implementation is much more complex for a space and time resolved mapping around a molten zone. We describe in this work a method based on interferometric imaging similar to shearing interferometers [15]. This scheme is based on the measurement of refractive index changes and makes under certain assumptions a two-dimensional, temporally resolved analysis of the temperature and pressure distribution possible.

\section{Experimental setup and methods}

\subsection{The setup}

The experimental setup is described in detail in [9]. Still, for a self-consistent description of the experiment a brief summary of the setup is given: The laser, running at $1064 \mathrm{~nm}$ with 10 ps FWHM duration pulses, is split into a pump and a probe beam. The pump beam is focused by an aspheric lens with NA 0.51 into a $1 \mathrm{~mm}$ thick D263 borosilicate glass sample through a polished facet on its long side $200 \mu \mathrm{m}$ below its surface (geometric distance) with $3.3 \mathrm{~W}$ average power after passing the lens (see Fig. 1).

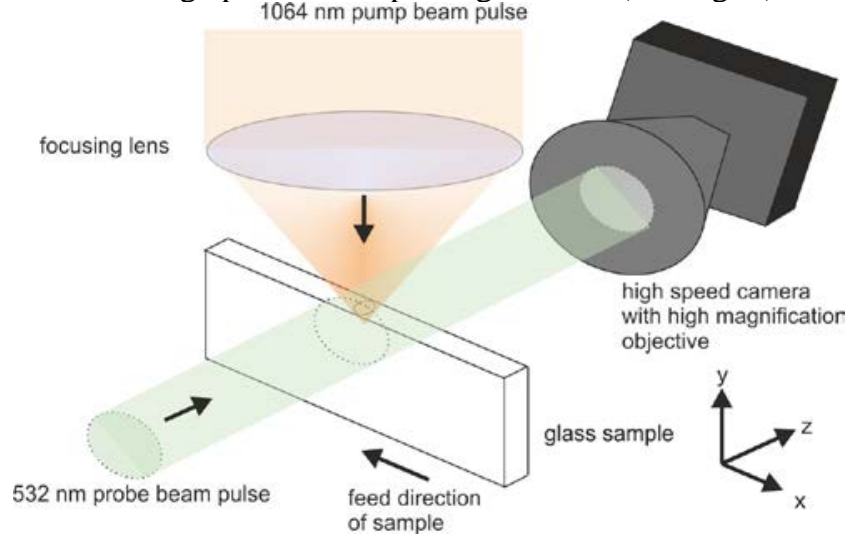

Fig. 1 Experimental setup schematics.

The probe beam is first frequency doubled, its diameter expanded, collimated and delayed in an adjustable delay line. Finally, the probe beam is launched through the pump interaction region (focal spot and molten zone) orthogonally to the pump beam and the moving direction of the sample. The probe beam transmitted through the interaction region is filtered by a laser line pass filter at $532 \mathrm{~nm}$ to remove thermal radiation and laser radiation from the pump beam and finally imaged by a high-speed camera synchronized to the laser repetition rate $(450 \mathrm{kHz})$ taking every second picture (camera frame rate $225 \mathrm{kHz}$ ). At the used magnification the nominal camera resolution lies at 2.13 $\mu \mathrm{m} /$ pixel while the optical resolution is limited to approximately $3 \mu \mathrm{m}$ by the camera objective's NA.

A typical example image with a resolution of $128 \times 128$ pixel provided by the setup is shown in Fig 2. Besides the obvious structures such as the bright edge of the elliptically shaped molten pool one can recognize also interference fringes. These interference fringes are caused by the double reflection of the 10 ps pulses on the glass surfaces: as the probe pulse passes the glass surface of the sample at the camera side a portion of this pulse is reflected back, hits the sample surface far from the camera, gets reflected there and this doubly-reflected pulse interferes with the original pulse at the camera sensor. Although the probe beam was collimated, due the variable delay line the beam waist did not coincide always with the sample's position. Also, the probe passed slightly off center through the sample area imaged by the high speed camera. Therefore, the visible fringe distribution is the interference result of two spherical waves that have propagated a different distance (twice the sample thickness).

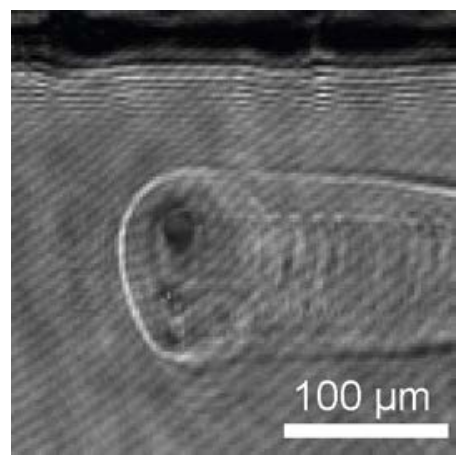

Fig. 2 Examplary camera image with the interference pattern. Feed speed $5 \mathrm{~mm} / \mathrm{s}$.

\subsection{Coherence considerations}

At first glance, this scenario with a twice reflected pulse interfering with the main pulse might look somewhat dubious, but it can be shown that this is indeed highly plausible when the pulse duration and glass sample thickness and surface reflectivities are considered:

- The reflectivity for normal incidence is given by the Fresnel equations and is due to the refractive index of 1.5265 for D263 glass at $532 \mathrm{~nm}$ (compare [16,17]) $4.3 \%$ per sample surface. Thus, the intensity of the twice reflected and transmitted beam is $0.19 \%$ of the directly transmitted beam. However, during interference the field amplitudes add up. Thus, the maximum achievable intensity modulation by interference in the here-discussed situation is $I_{0} \cdot(1 \pm 0.043)^{2}$.

- The time delay introduced by a $1.1 \mathrm{~mm}$ thick D263 borosilicate glass plate is given by twice the glass plate thickness and the group velocity $v_{G}$ of the pulse, given by $\partial \omega / \partial k$ with $\omega$ and $k$ being the angular frequency and the wave vector of light propagating through the glass which gives effectively $v_{G}=c_{0} / n$, with $c_{0}$ the speed of light in vacuum and $n$ the refractive index of the glass (see above). Thus, the time delay amounts to $11.2 \mathrm{ps,}$ while the used laser pulses have a FWHM pulse duration of 10 ps. Assuming a Gaussian pulse shape, there is an effective pulse overlap between both pulses present in the camera.

- Considering both above effects to calculate the time integrated recorded intensity by the camera it is found that in case of destructive respectively constructive in- 
terference the intensity is reduced respectively increased by $3.7 \%$ with respect to the average intensity of the directly transmitted pulse. Assuming that the average intensity is set to achieve about $50 \%$ gray level on the image, the achievable image brightness modulation should be on the order of 7-8 gray levels out of 255 for a linear camera transfer characteristic.

Fig. 3 shows the pixel gray values of a typical camera image with the observed interference fringes. As can be seen, the modulation depth is with up to 40 gray level modulation depth actually larger than expected by the calculation. There are several possibilities which might increase the observed modulation depth: the pulse shape itself might be different than a Gaussian - e.g. a hyperbolic secant pulse would lead to larger modulation even at the same FWHM pulse duration. Also the effect of the laser line filter for the probe beam at $532 \mathrm{~nm}$ as a bandpass filter, as well as other dispersive mechanisms that would increase the pulse duration of the probe pulses and thereby increase the interference fringe modulation depth were not considered in the above discussion.

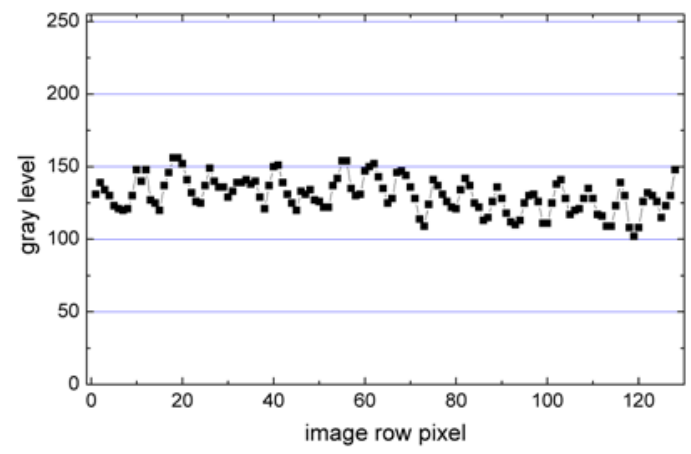

Fig. 3 Modulation depth of interference fringes taken from a typical high speed camera image.

\subsection{Calculation of phase information}

The following section describes the evaluation method to extract the phase information from the camera images (compare Fig. 4): Each image was processed by the program IDEA 1.7 (Interferometric Data Evaluation Algorithms), see $[18,19]$.
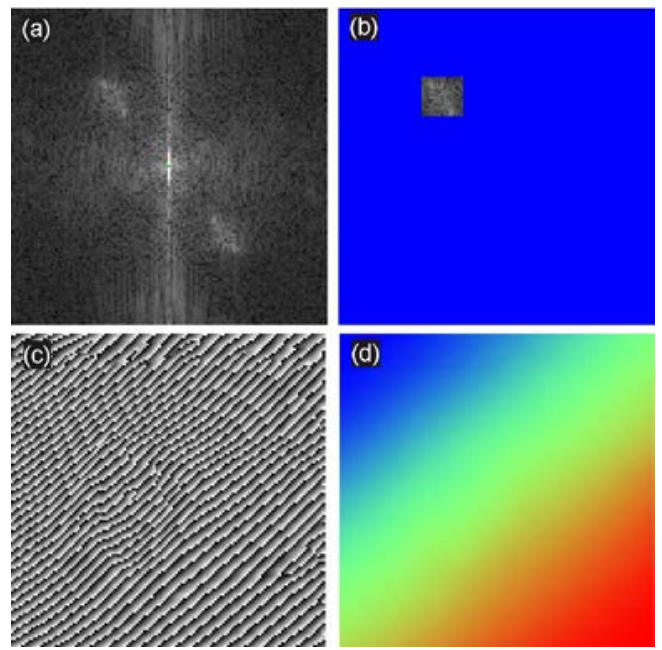

Fig. 4 Fringe pattern to phase data calculation process using as input image Fig. 2. (a) 2D Fourier transform, (b) masking of relevant spatial frequency region, (c) wrapped phase of the backward Fourier transformation, (d) DCT unwrapped phase
First, a 2D Fourier transform was applied to the images giving a spectrogram of the spatial frequency components. The periodicity of the interference fringes provides an increased signal level, i.e. the Fourier-transformed signal of the interference pattern signal can be easily distinguished, at certain spatial frequencies. Selecting this frequency range, i.e. setting all other values of the spectrogram by a mask to zero, the inverse Fourier transformation provides the phase information, with phase values running from $-\pi$ to $\pi$. The resulting phase data were unwrapped by discrete cosine transformation (DCT) to give a smooth phase distribution of the image. As can be expected from the fringe pattern (e.g. compare Fig. 2) the unwrapped phase has a quite a strong slope. In order to get rid of this slope the phase run of the first image was subtracted from every subsequent phase image. This way, the process relevant phase changes can be made visible, because at the very start of the processing the glass is unchanged yet, so one can assume that there are no phase changes present. An example is shown in Fig. 5.

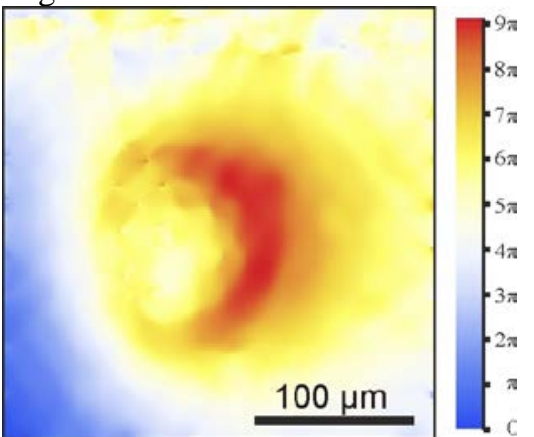

Fig. 5 Phase changes after $89 \mathrm{~ms}$ at a feed speed of $5 \mathrm{~mm} / \mathrm{s}$, compare Fig. 2.

Since the DCT phase unwrapping algorithm requires a known starting phase at a certain point, we used the bottom-left corner pixel to have a phase of 0 for every unwrapping. However, considering that phase differences are calculated between the subsequent and the first image a phase shift of the bottom left corner pixel during the process would lead to incorrectly calculated phases. To prevent this, we tracked the unwrapped phase of the bottom-left corner pixel across the whole video sequence and added to each phase-unwrapped frame the tracked bottom-left corner pixel phase value. We also made sure, whether or not the phase of the bottom-left corner pixel needs to be unwrapped across the whole video sequence by observing the movement of the interference pattern: in case the interference pattern around the pixel in question moves further than one stripe phase unwrapping becomes necessary.

\subsection{Investigated parameters}

During the experiment the laser pulse energy and repetition rate were kept constant at $7.3 \mu \mathrm{J}$ and $450 \mathrm{kHz}$. The feed speed was varied between 2, 5, 10, 20, 50, 100 and $200 \mathrm{~mm} / \mathrm{s}$. During the experiment described in [13] variations of the probe pulse time delay were conducted, but we limit us here mainly to video sequences at which the probe delay was 9.6 ns. Only one data set is shown here at which the delay was 3 ns. However, because the overall increase of the refractive index can be assumed to be caused by thermodynamical effects that occur on relatively long timescales unlike plasma motion or shockwave generation 
(which will be described elsewhere), we consider the actual setting of the probe-pulse timing delay not to have a significant effect on the observed phase changes.

\subsection{Phase comparison of unmodified glass, hot and cold welding seam}

In one of the experiments we imaged unmodified glass with only a single pulse arriving in the focal area, then after processing for $100 \mathrm{~ms}$ at a feed speed of $5 \mathrm{~mm} / \mathrm{s}$ (probe delay $3 \mathrm{~ns}$ ). Finally, after a cool down period of several minutes the last position was imaged by the same camera without the pump beam present. Using the method described in section 2.3, the phase changes of the hot and cooled down welding seam were calculated by subtracting the phase of the initial frame.

\subsection{Calculation of 3D phase distribution}

For the calculation of a 3D phase distribution a single camera as used here is typically not sufficient. Only under certain assumptions such as rotational or cylindrical symmetry a three-dimensional phase distribution can be derived by using inverse Abel transformation or inverse Radon transformation. Strictly speaking, the geometry of the welding seam, see Fig. 6, is not cylindrically symmetric. However, an approximate cylindrical symmetry in the halfspace after the seam (in Fig. 6 to the left) can be made out for feed speeds of 5 and $2 \mathrm{~mm} / \mathrm{s}$.

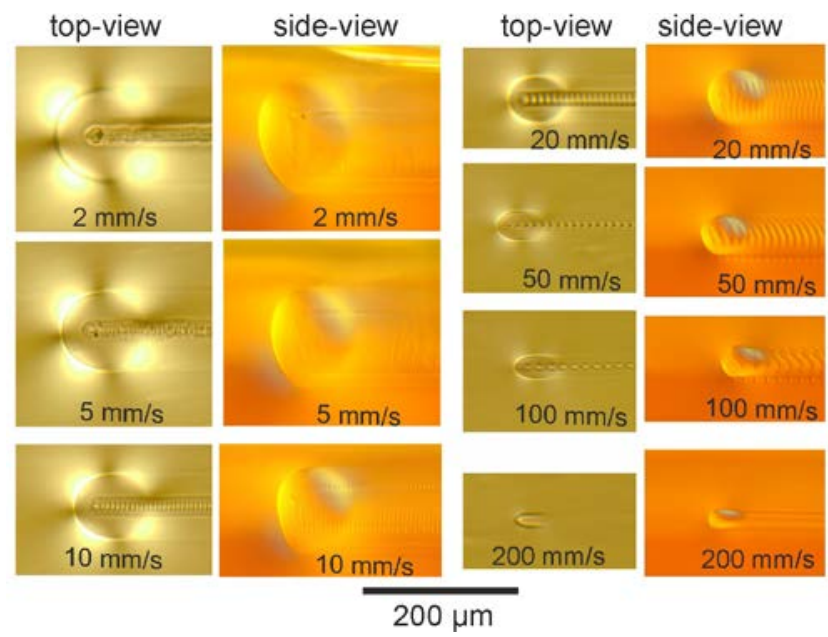

Fig. 6 Top and side views of the end points of the molten zones at different feed. The scale bar is valid for all images, the laser arrives for side-view images from the top.

For these feed speeds it is possible to calculate an approximate phase distribution in the half space of unmodified glass by assuming that the cylinder axis runs just directly from bottom tip to the uppermost point of the welding seam in the side view. To create the data for the reconstruction of the phase distribution we took for each pixel row of the last frame the phase values of pixels from the left image border up to the cylinder axis going through the tear drop shape of the seam. This phase run was then mirrored at the axis resulting in a symmetric phase function for each row. Each such symmetrized row can now be considered as an integrated view of the phase distribution at this image height (row) into the depth (say z-direction, if the camera image width is defined as " $x$ " and its height as " $y$ "direction - see Fig. 1) seen from the current (and only one) angle of the camera, see Fig. 7. We can consider this phase run to be the result of an Abel or Radon transformation for a single angle. However, assuming that the phase distribution is cylindrically symmetric, we would expect the same phase run independent on the actual viewing angle. Therefore, it is possible to construct under this assumption a (hypothetical) 2D radon image of this phase run, simply by stacking it for each hypothetical angle under which this circularly symmetric y-z-phase distribution was observed (which is the same as the radon transformation). The inverse Radon transformation gives then the circularly symmetric phase distribution of the row. Since the procedure can be done for every row of the phase image it is possible to construct a 3D phase distribution. Of course the validity of this distribution is only limited to the unmodified halfspace of glass in front of the seam, but it provides at least some qualitative values of the thermomechanical glass state in front of the welding seam during welding. Please note, that resulting units of the inverse Radon transformation is a phase density (i.e. per voxel), with the dimensions of the voxel given in $x-y$ direction by the pixel dimensions of a row image and the glass thickness, while in the z-direction the voxel size has the same size as the original image resolution.

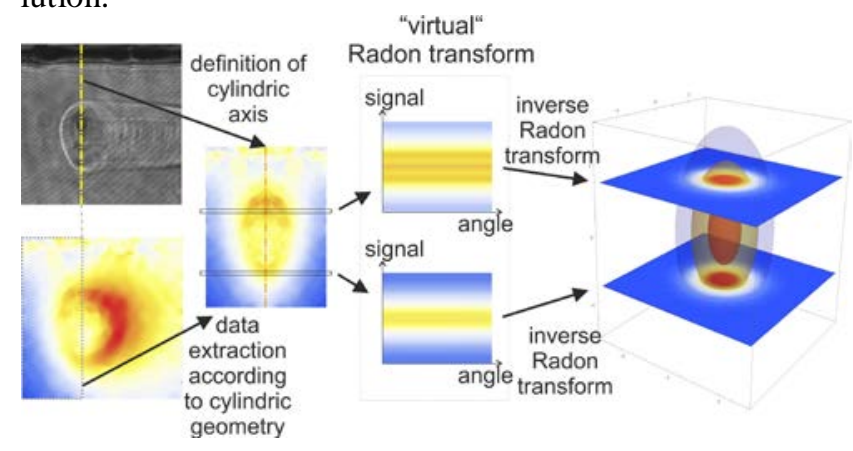

Fig. 7 Schematic reconstruction process of the 3D phase distribution, assuming cylindrical symmetry of welding seam.

\subsection{Derivation of physical quantities from the 3D phase distribution}

It is known that the refractive index of most glass types depends on the applied stress or temperature. Therefore it is possible to calculate these quantities, if the phase distribution (effectively the spatial phase density, see above) is known. Assuming the refractive index change $\Delta n$ is a monotonic function depending on the physical property $\Psi$ (i.e. temperature or pressure) the corresponding phase change $\Delta \varphi$ can be expressed as

$$
\Delta \varphi=\Delta n(\Psi) \cdot k_{0} x=\left(\frac{2 \pi}{\frac{\lambda_{0}}{n_{0}+\Delta n(\Psi)}}-\frac{2 \pi}{\frac{\lambda_{0}}{n_{0}}}\right) x
$$

with $\lambda_{0}$ the wavelength of light in vacuum, $k_{0}$ the corresponding wave number in vacuum, $n_{0}$ the refractive index of the glass at normal conditions and $x$ the voxel size in $\mathrm{x}$ direction (or y-direction). For an assumed linear dependence of $\Delta n$ on $\Psi$, one arrives at

$\Psi=\Delta \varphi /\left(c \cdot k_{0} \cdot x\right)$ 


\section{Results}

Due to the length limitations only a selection of results can be shown. We start first with a comparison of the timedependent phase evolution for different feed speeds at certain image points that have a fixed distance to the focal spot of the laser. Also the maximum and minimal phase for each frame is shown. The chosen points are shown in Fig. 8.
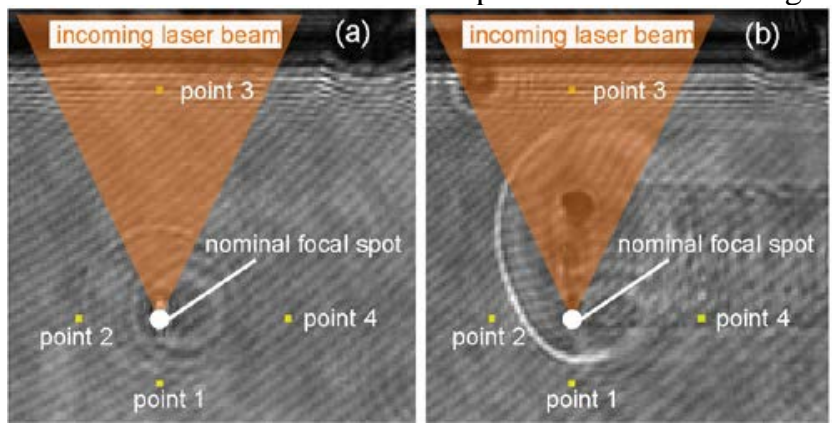

Fig. 8 Layout of the points at which the time-dependent phase evolution is tracked. (a) shows the beginning of the welding, (b) shows the state of the molten zone at the last frame after $222 \mathrm{~ms}$ at a feed speed of $2 \mathrm{~mm} / \mathrm{s}$

The reasoning for their placement is as follows: all points are chosen such that they always lie outside the highly dynamic molten zone even for the slowest feed speed. Point 1 measures the phase changes $42.5 \mu \mathrm{m}$ below the nominal focal spot and since the temperature distribution inside the melt is very likely inhomogeneous (see [8]), point 3 which lies $149 \mu \mathrm{m}$ above the focal spot can be used to compare the phase evolution with respect to point 1 . Point 2 is used to detect the phase evolution ahead of the welding seam $(-53.2 \mu \mathrm{m}$ ahead of the focal spot), while point 4 measures the phase evolution in the solidification area $85.1 \mu \mathrm{m}$ after the focal spot. The minimum and maximum phase values are useful to put these selected points into perspective in comparison to the whole phase evolution during the process.

The results are shown in Fig. 9. Clearly, for lower feed speeds there is a significant phase rise over time, obviously because the molten zone becomes bigger and hotter. For feed speeds above $50 \mathrm{~mm} / \mathrm{s}$ even the maximum phase (of the whole image) does not increase significantly, which is why we do not show here the results for $200 \mathrm{~mm} / \mathrm{s}$ feed speed as they are very similar to the $100 \mathrm{~mm} / \mathrm{s}$ results. For some time positions, phase jumps about $1 \pi$ (and back) can be observed. This is due to the noise inherent in image acquisition and phase calculation. Indeed, the bottom line or background of each phase run represents the true phase evolution best. The reader might note one further puzzling point, which are the phase runs of certain points starting sometimes above and sometimes below zero phase. This is just a computational artefact of using the first image as the phase reference: since there is also noise present it will determine the starting value of all phase runs in a random way.

We would like to point out that we didn't show the phase run of a point from inside the melt pool because inside the melt pool the pressure, temperature and plasma can influence the refractive index in different directions, i.e. increasing or decreasing it. Therefore the resulting phase is something of an average across these different zones. This explains the reduced phase values inside the melt zone visible in Fig. 5 or Fig. $10(100 \mathrm{~mm} / \mathrm{s})$. In contrast, the process for calculating the 3D phase distribution is able to infer the phase values also inside the molten zone, even if it is only a rough estimate, due to the used approximations.
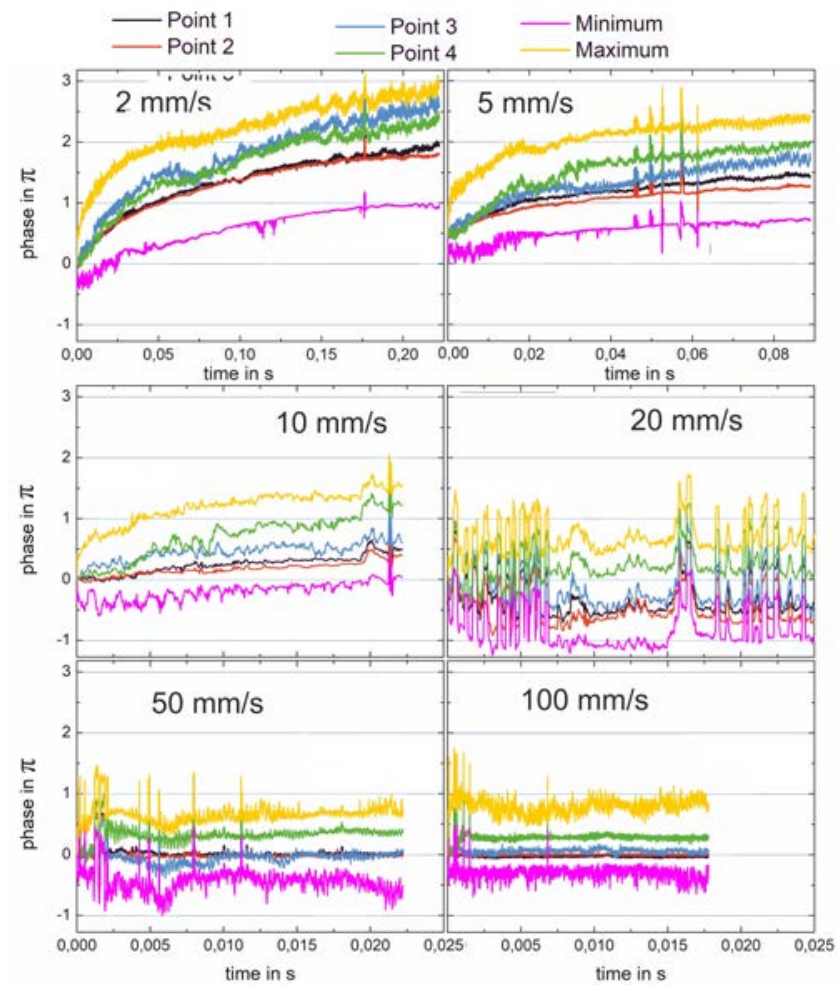

Fig. 9 Time dependent phase evolution at points 1-4 (see Fig. 8) and of the phase maxima and minima at different feed speeds. Please note the different time scales at 2 and $5 \mathrm{~mm} / \mathrm{s}$.

Fig. 10 shows the phase evolution for all investigated feed speeds at $10 \mathrm{~ms}$ after starting the process. The phase scale bars are set up such that they encompass the lowest and highest measured phase of the whole image sequence, thus the maximum phases might not be necessarily reached in Fig. 10 as only a single phase frame is shown. Clearly, the laser interaction with glass creates very steep phase slopes at the edges at which the molten zone is expected. However, the phase increases also in the vicinity of the molten zone. Moreover, even at this short time interval already the effects of the feed direction and the feed speed are visible, because to the left of the molten zone the phases are smaller, while to the right higher phases are achieved (the glass plate moves from left to right). This happens partly due to the effective heat accumulation but also due to the refractive index change of the cooling, laser modified region to the right (see 100 and $200 \mathrm{~mm} / \mathrm{s}$ feed speeds). To better observe the time evolution of the 2D phase distribution please consult [20] which provides online the video sequences (10x time-lapse) of the calculated phases.

The results for $20 \mathrm{~mm} / \mathrm{s}$ are different from the rest (see also Fig. 9) and are likely due to the presence of an accidental fluctuation in the initial frame that is used as a reference frame for calculating the phase difference (see section 2.3). A comparison using the corresponding video in [20] $(20 \mathrm{~mm} / \mathrm{s})$ shows that the structure of the frame shown in Fig. 10 acts as an overlay on a structure that is similar to the phase evolution of 10 and $50 \mathrm{~mm} / \mathrm{s}$. 

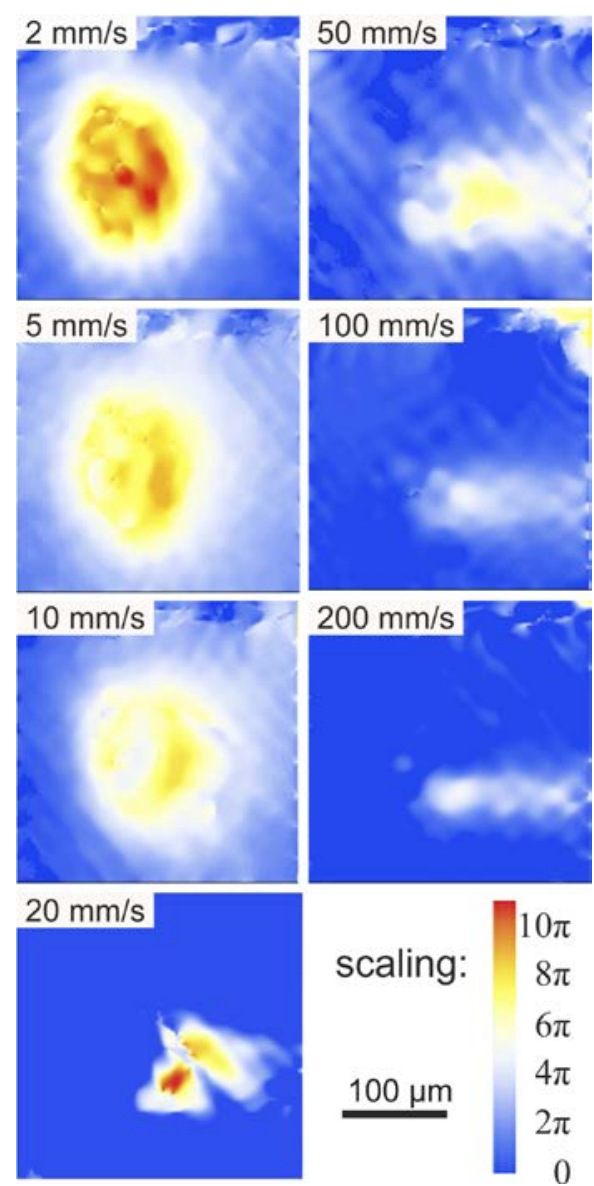

Fig. 10 Phase distributions for different feed speeds at $10 \mathrm{~ms}$ after starting the irradiation.

\subsection{Phase of the hot and cold welding seam}

Fig. 11 gives the comparison between phase distributions for the same laser modified region during the irradiation process and after cool down to room temperature as described in section 2.5. The black region in the phase distributions corresponds to phase value of zero. Clearly, the cooled down laser modified zone provides a weak lensing effect with a smaller-than-average refractive index. The area surrounding the cold modified zone, i.e. outside the black contour, exhibits phase values between $0.5 \pi$ and $1 \pi$. Consequently, here is the refractive index higher than in unmodified glass.

Both effects - the reduction of refractive index inside the modified zone and its increase outside - can be likely explained as stress induced birefringence where the birefringence is induced by the laser modified zone into the surrounding glass, as it is known that the laser modified zone of D263 glass experiences under similar processing parameters a volume increase [21]. In contrast, the hot molten pool shows a significantly increased phase (up to $4.5 \pi$ ). The increase of the refractive index extends also beyond the edges of the molten pool.

Of course, during the hot state temperature and stress induced refractive index changes will coexist, while, at first glance, there should be only stress induced changes in the cold state. Unfortunately, the situation is not quite as simple as to be able to exactly determine the temperature-only induced refractive index changes by subtracting the cold phase distribution from the hot one. The reasons are on one hand that in the hot state the pressures are higher and on the other hand chemical modifications of the glass network occur at such high temperatures and pressures and have a direct impact on the refractive index [22]. Nonetheless, such a consideration will provide an upper limit on the temperature-only caused changes of the refractive index and is shown in Fig. 12 (a). Following the method described in section 2.6 its symmetrized approximate threedimensional phase distribution is shown in Fig. 12 (b).

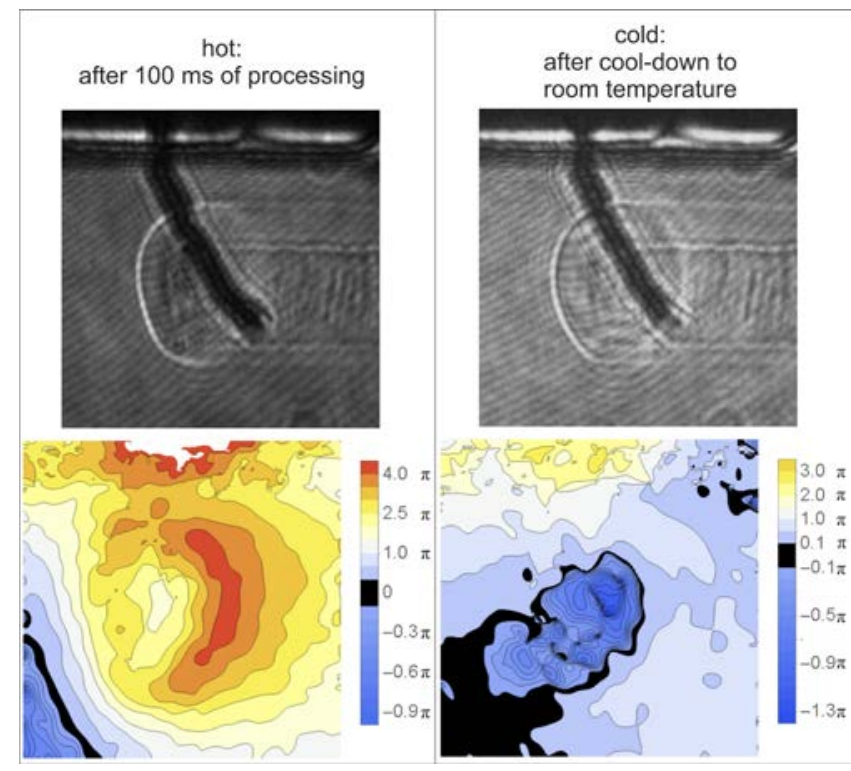

Fig. 11 Image frames and corresponding phase distributions for a feed speed of $5 \mathrm{~mm} / \mathrm{s}$. Please note that the colors have a different scaling above and below zero. Also due to different minimum and maximum valueas the zero value lies in different color regions
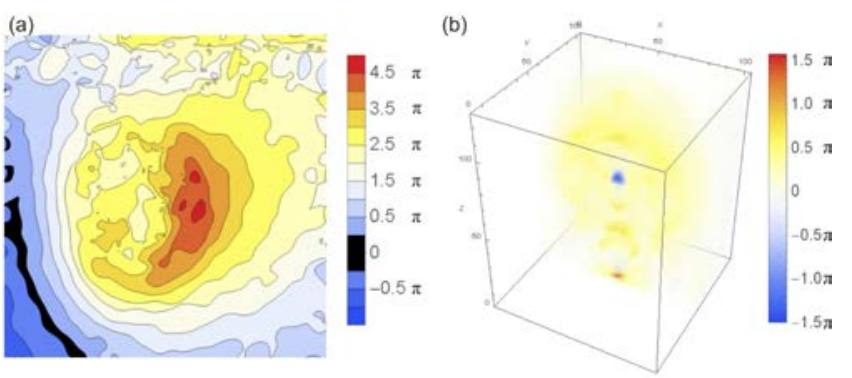

Fig. 12 (a) upper limit for temperature-only caused phase changes; (b) resulting approximate threedimensional phase distribution under assumption of cylindrical symmetry; the unit is phase per voxel with a voxel size of $9.6 \times 9.6 \times 2.1 \mu \mathrm{m}^{3}$.

Similarly, if material densification effects are neglected and therefore all induced phase changes can be considered to be caused by mechanical stress due to volume expansion of the molten zone, the phase distribution of the cold modified welding seam (see Fig. 11) can be also interpreted approximately as the upper limit of the induced phase changes by mechanical stress. The corresponding threedimensional phase distribution is shown in Fig. 13.

Finally, the results of the three-dimensional phase distributions for the upper limits of phase changes induced by thermal or mechanical action can be used to estimate the maximum temperature during the process and the maximum stress distribution of the cold, laser modified area 
(and its vicinity). To get these physical quantities the knowledge of the coefficients of temperature-dependent and stress-dependent refractive index changes is necessary. The stress optical coefficient for D263 glass [23] is $3.47 \times 10^{-12} \mathrm{~m}^{2} / \mathrm{N}$. Unfortunately, the thermal coefficient of D263 glass is not available. Since D263 is nonetheless a borosilicate glass we chose to substitute in this work the thermal coefficient of $\mathrm{N}-\mathrm{BK} 7$ glass to use as a calculation example. The name N-BK7 stands for borosilicate crown glass and has a thermal coefficient close to $3 \times 10^{-6} 1 / \mathrm{K}$ [24] (the literature gives the value of $2.8 \times 10^{-6} 1 / \mathrm{K}$ for $546 \mathrm{~nm}$ wavelength at $140{ }^{\circ} \mathrm{C}$ ).

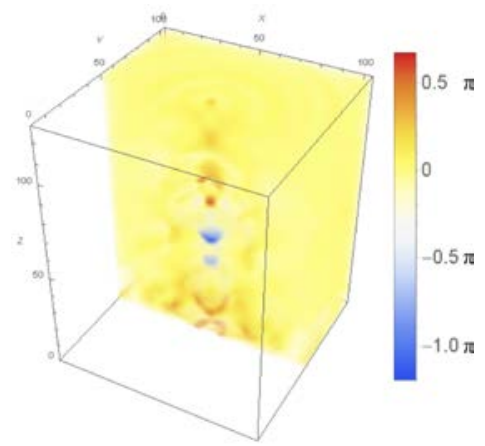

Fig. 13 Approximate upper limit on the three-dimensional phase distribution resulting from mechanical stress of a cold laser modified zone. ; the unit is phase per voxel with a voxel size of $9.6 \times 9.6 \times 2.1 \mu^{3}$.

Following section 2.7 the above coefficients give for the temperature distribution a value of $8400 \mathrm{~K}$ per $\pi$ for the phase distribution in Fig. 12 (b) and a stress of 7.2 GPa per $\pi$ for the Fig. 13. While the maximum calculated temperature clearly exceeds by more than an order of magnitude the melting temperature of D263, the chosen coefficient was only an example to demonstrate the calculation process. It is also necessary to mention that due to the symmetrization process of the data there will be discontinuities at the cylindrical axis which are hard to handle for the inverse Radon transformation. Indeed, the highest and lowest values lie exactly on this axis. Furthermore, in order to be able to predict the temperatures around the molten zone it is necessary to be sure that the thermo-optic coefficient is a monotonic, ideally linear, function of the temperature. Currently we propose that the unrealistic temperature determined is indeed a result of the nonlinear dependency of the refractive index with temperature which was observed in borosilicates [26]. This emphasizes that a better knowledge of the variation of the refractive index in both temperature and pressure is needed to properly in estimate Fig 12 (b).

The observed values for the stress, at least off-axis, seem to be more realistic. The tensile strength of D263 is with $72.9 \mathrm{GPa}$ [27] approximately 10 times as large as the lowest value, but on the same order of magnitude that regular glasses [28]. Here again the numbers need to be taken with care since it is well known than the refractive index is sensitive not only to the residual stress but also to the cooling rate [29].

\section{Conclusion and outlook}

In this work we investigated the phase changes induced by ultra-short pulsed processing of glass as is often the case in ultra-short pulsed glass welding. We have shown that gradual phase changes can be observed and tracked across time durations of several $100 \mathrm{~ms}$. Furthermore, the geometry of the laser induced modifications can be used to roughly estimate the three-dimensional phase distribution which can be used in principle to estimate the temperature and pressure distributions in the surrounding area effectively on a pulse-by-pulse basis. Comparisons of the hot material with the cooled area allow furthermore to determine an upper limit on the achieved temperature and pressure distribution. While for the induced mechanical stress plausible values have been achieved, the method did not work out for the temperature, mainly due to the lack of valid thermooptic coefficient for this glass type and temperature range. Future investigations on laser induced glass modifications will be necessary to confirm the validity of this approach for determining the temperature and stress distributions.

\section{Acknowledgments and Appendixes}

The authors gratefully acknowledge support by the Deutsche Forschungsgemeinschaft through the grant No. Ho1691/8-1 | Li2713/4-1 | Schm2115/62-1 and the Graduate School in Advanced Optical Technologies (SAOT) of the Friedrich-Alexander-University of Erlangen- Nürnberg.

\section{References}

[1] M. Berndt, and M. Danner: LTJ, 50, (2016) 42.

[2] J. Gottmann, M. Hermans, N. Repiev, and J. Ortmann: Micromachines, 8, (2017) 110.

[3] T. Tamaki, W. Watanabe, and K. Itoh: Opt. Express, 14, (2006) 10460.

[4] M. J. Weber: "Handbook of Optical Materials", (CRC Press, Boca Raton, 2002), pp.261.

[5] T. Topcu, and F. Robicheaux: Phys. Rev. A, 86, (2012) 053407.

[6] J.-C. Diels, W. Rudolph: "Ultrashort Laser Pulse Phenomena“, ed. by P. Liao and P. Kelley, (Elsevier Inc., Amsterdam, 2006), pp.205.

[7] M. Sun, U. Eppelt, S. Russ, C. Hartmann, C. Siebert, J. Zhu, W. Schulz: Opt. Express, 21, (2013) 7858.

[8] I. Miyamoto, K. Cvecek, Y. Okamoto, M. Schmidt: Appl. Phys. A, 114, (2013) 187.

[9] R. Poprawe: „Lasertechnik für die Fertigung“, (Springer-Verlag, Berlin, 2005), p. 142.

[10] S. Pfalzner: "An Introduction to Inertial Confinement Fusion”, ed. by S. Cowley, (Taylor \& Francis Group, Boca Raton, 2006), pp.73.

[11] I. Miyamoto, Y. Okamoto, R. Tanabe, Y. Ito, K. Cvecek and M. Schmidt: Opt. Express 24, (2016) 25718.

[12] T. Makabe, Z. L. Petrovic: "Plasma Electronics: Applications in Microelectronic Device Fabrication", (Taylor \& Francis Group, Boca Raton, 2006), pp. 30.

[13] I. Alexeev, J. Heberle, K. Cvecek, K.Y. Nagulin, and M. Schmidt: Micromachines 6, (2015) 1914.

[14] M. Matsumoto, K. Wada, Y. Ozeki and K. Itoh, Proc. of LAMP2009 - the 5th International Congress on Laser Advanced Materials Processing, Kobe, Japan, 2009

[15] M. E. Riley and M. A. Gusinow: Appl. Opt. 16, (1977) 2753.

[16] https://refractiveindex.info/?shelf=glass\&book=SCHO TT-multipurpose\&page=D263TECO 
[17] https://refractiveindex.info/download/data/2017/schott _2017-01-20b.agf

[18] http://www.optics.tugraz.at/idea/idea.html

[19] M. Hipp, J. Woisetschlaeger, P. Reiterer, and T. Neger Digital evaluation of interferograms, Measurement, 36, (2004) 53.

[20] http://dx.doi.org/10.17632/drx34gv4kc.1

[21] K. Cvecek, R. Odato, S. Dehmel, I. Miyamoto, and M. Schmidt: Opt. Express, 23, (2015) 5681.

[22] R.M. Waxler and G.W. Cleek: Natl. Bur. Stand. Sec. A 77A, (1973) 755.

[23] https://www.shibuyaopt.co.jp/window/PCP_D263Teco_character.pdf

[24] https://www.schott.com/d/advanced_optics/3794edededd2-461d-aec5-0a1d2dc9c523/1.0/schott_tie19_temperature_coefficient_of_refractive_index_eng.p df

[25] https://www.schott.com/nexterion/english/application/f aq/general.html

[26] L. Prod'homm: Phys. Chem. Glasses, 1, (1960) 119.

[27] http://heraeusquarzglas.de/en/quarzglas/mechanicalpro perties/Mechanical_properties.aspx

[28] K. J. Kasunic, “Optomechanical Systems Engineering”, ed. by G. Boreman, (Wiley, Hoboken, 2015), pp. 119.

[29] J. E. Shelby, "Introduction to Glass Science and Technology”, (The Royal Society of Chemistry, Cambrige, 2005), p. 204.

(Received: June 19, 2018, Accepted: November 25, 2018) 